\title{
Passivity and Synchronization of Coupled Complex-Valued Memristive Neural Networks*
}

\author{
$1^{\text {st }}$ Yanli Huang, $2^{\text {nd }}$ Jie Hou \\ Tianjin Key Laboratory of Optoelectronic \\ Detection Technology and System \\ School of Computer Science and Technology \\ Tiangong University \\ Tianjin 300387, China \\ huangyanli@tjpu.edu.cn, JHou_TJPU@163.com
}

\author{
$3^{\text {rd }}$ Shunyan Ren \\ School of Mechanical \\ Engineering \\ Tiangong University \\ Tianjin 300387, China \\ renshunyan@163.com
}

\author{
$4^{\text {th }}$ Erfu Yang \\ Department of Design, Manufacture \\ and Engineering Management \\ Faculty of Engineering \\ University of Strathclyde \\ Glasgow G1 1XJ, Scotland, UK \\ erfu.yang@strath.ac.uk
}

\begin{abstract}
The coupled complex-valued memristive neural networks (CCVMNNs) are investigated in this study. First, we analyze the passivity of the proposed network model by designing an appropriate controller and using certain inequalities as well as Lyapunov functional method, and provide a passivity condition for the considered CCVMNNs. In addition, a criterion for guaranteeing synchronization of this kind of network is established. Finally, the effectiveness and correctness of the acquired theoretical results are verified by a numerical example.
\end{abstract}

Index Terms-memristive neural networks, synchronization, passivity, state coupling

\section{INTRODUCTION}

Recently, coupled neural networks (CNNs) have been widely concerned owning to their extensive application in secure communication, chaos generator design, brain science, etc. As we all know, these applications heavily depend on the dynamic behaviors of CNNs, especially the synchronization and passivity of CNNs [1]-[5]. In [2], the impulsive synchronization of Markovian jumping randomly CNNs was considered by using multiple integral approach. Some conditions for guaranteeing synchronization of CNNs were obtained in [3]. Ren et al. [5] analyzed the passivity of CNNs with directed and undirected topologies.

In 1971, Chua [6] first proposed the concept of memristor. Unlike resistor, because the memristance depends on the amount of charge passing through it, the memristor can remember its past dynamic history. Therefore, the memristor is widespread used in signal processing as well as device modeling, especially in simulating synaptic behavior [7]-[11]. Moreover, memristive neural networks (MNNs) can better present the neural processes in the human brain [12]. In recent years, coupled memristive neural networks (CMNNs) have been extensively studied and numerous interesting studies on

This work was supported in part by the Natural Science Foundation of Tianjin City under Grant 18JCQNJC74300 and in part by China Scholarship Council (No.201808120044). Dr Yang is supported in part by the Advanced Forming Research Centre (AFRC) and Lightweight Manufacturing Centre (LMC) under the Route to Impact Programme 2019-2020 funded by Innovate UK High Value Manufacturing Catapult [grant no.: AFRC_CATP_1469_R2IAcademy].
CMNNs have been reported [13]-[15]. In [13], the robust synchronization of CMNNs with uncertain parameters was discussed.

In fact, complex-valued neural networks (CVNNs) are extensions of real-valued neural networks in which the states, connection weights as well as activation functions are all complex-valued. Certain practical problems cannot be solved by real-valued neural networks but can be better solved with CVNNs. In addition, CVNNs have a wide range of applications, including emotion analysis, analogy amplification, computer version, imaging, etc. Hence, a large number of studies have been conducted on the dynamic behavior of CVNNs [16]-[19]. Complex-valued MNNs (CVMNNs) can be also built by replacing resistors with memristor in VLSI circuits of CVNNs as described in [20], which is widely applied in image processing, engineering optimization and pattern recognition. Therefore, it is meaningful to study the passivity and synchronization in CVMNNs [20]-[23]. The authors in [20] considered the exponential stability of CVMNNs. The synchronization of uncertain fractional-order CVMNNs with multiple time delays was analyzed in [21]. To the best of our knowledge, the passivity and synchronization of coupled CVMNNs (CCVMNNs) has not yet been studied.

Accordingly, the principal goal in the present study is to investigate the passivity and synchronization problems of CCVMNNs. Firstly, a criterion for ensuring passivity of CCVMNNs is put forward by constructing an appropriate controller and using Lyapunov functional method. Secondly, we also establish a synchronization condition for the considered network. Finally, a numerical simulation is given to verify the correctness of the results.

\section{PRELIMINARIES}

$\mathbb{R}^{N}$ and $\mathbb{C}^{N}$ respectively symbol the $N$-dimensional real vector space and the $N$-dimensional complex vector space. $\lambda_{m}(\cdot), \lambda_{M}(\cdot)$ denote the minimal and the maximal eigenvalue of the corresponding matrix. Let $e=e^{R}+i e^{I}$ be a complex number, where $i$ symbols the imaginary unit, which satisfies $i=\sqrt{-1}$ and $e^{R}, e^{I} \in \mathbb{R}$ are the real and imaginary part of $e$. The norm in $\mathbb{C}^{N}$ is denoted as $\|\cdot\|$. For any vector 
$e(t) \in \mathbb{C}^{N},\|e(t)\|=\sqrt{e^{H}(t) e(t)}$ where $H$ denotes the conjugate transposition. Let $e^{R}(t), e^{I}(t) \in \mathbb{R}^{N}$ be the real and imaginary part of $e(t) \in \mathbb{C}^{N}$, then one has $\|e(t)\|=$ $\sqrt{\left(e^{R}(t)\right)^{T} e^{R}(t)+\left(e^{I}(t)\right)^{T} e^{I}(t)}$.

\section{PASSIVITY AND SYNCHRONIZATION OF CCVMNNS}

\section{A. Network model}

On the basis of the physical characteristics of memristor, a single CVMNN model can be described by

$$
\begin{aligned}
\dot{z}_{\iota}(t)= & -a_{\iota} z_{\iota}(t)+\sum_{j=1}^{n} b_{\iota j}\left(z_{\iota}(t)\right) h_{j}\left(z_{j}\left(t-\tau_{j}(t)\right)\right) \\
& +\sum_{j=1}^{n} d_{\iota j}\left(z_{\iota}(t)\right) f_{j}\left(z_{j}(t)\right), \iota=1,2, \cdots, n
\end{aligned}
$$

where $z_{\iota}(t)$ denotes the complex-valued state variable of $\iota$-th neuron. $a_{\iota}>0$ is the self-inhibition. $b_{\iota j}\left(z_{\iota}(t)\right)$ and $d_{\iota j}\left(z_{\iota}(t)\right)$ represent complex-valued memristors synaptic connection weights. $h_{j}(\cdot)$ and $f_{j}(\cdot)$ stand for complex-valued activation functions for the delayed configuration and nondelayed one of the $j$-th neuron. The time-varying delay $\tau_{j}(t)$ satisfies $0 \leqslant \tau_{j}(t) \leqslant \tau_{j} \leqslant \tau=\max _{j=1,2, \cdots, n}\left\{\tau_{j}\right\}, \dot{\tau}_{j}(t) \leqslant \gamma_{j}<1$.

Let $z_{\iota}(t), b_{\iota j}\left(z_{\iota}(t)\right), d_{\iota j}\left(z_{\iota}(t)\right), h_{j}(\cdot)$, and $f_{j}(\cdot)$ be the following:

$$
\begin{aligned}
z_{\iota}(t) & =z_{\iota}^{R}(t)+i z_{\iota}^{I}(t), \\
b_{\iota j}\left(z_{\iota}(t)\right) & =b_{\iota j}^{R}\left(z_{\iota}^{R}(t)\right)+i b_{\iota j}^{I}\left(z_{\iota}^{I}(t)\right), \\
d_{\iota j}\left(z_{\iota}(t)\right) & =d_{\iota j}^{R}\left(z_{\iota}^{R}(t)\right)+i d_{\iota j}^{I}\left(z_{\iota}^{I}(t)\right), \\
f_{j}\left(z_{j}(t)\right) & =f_{j}^{R}\left(z_{j}^{R}(t)\right)+i f_{j}^{I}\left(z_{j}^{I}(t)\right), \\
h_{j}\left(z_{j}\left(t-\tau_{j}(t)\right)\right) & =h_{j}^{R}\left(z_{j}^{R}\left(t-\tau_{j}(t)\right)\right)+i h_{j}^{I}\left(z_{j}^{I}\left(t-\tau_{j}(t)\right)\right),
\end{aligned}
$$

where $z_{\iota}^{R}(t), b_{\iota j}^{R}\left(z_{\iota}^{R}(t)\right), d_{\iota j}^{R}\left(z_{\iota}^{R}(t)\right), f_{j}^{R}\left(z_{j}^{R}(t)\right), h_{j}^{R}\left(z_{j}^{R}(t-\right.$ $\left.\tau_{j}(t)\right)$ are the real parts of $z_{\iota}(t), \quad b_{\iota j}\left(z_{\iota}(t)\right), \quad d_{\iota j}\left(z_{\iota}(t)\right), f_{j}\left(z_{j}(t)\right), h_{j}\left(z_{j}\left(t-\tau_{j}(t)\right)\right)$, respectively. $i$ is the imaginary unit which satisfies $i=\sqrt{-1}$. $z_{\iota}^{I}(t), \quad b_{\iota j}^{I}\left(z_{\iota}^{I}(t)\right), \quad d_{\iota j}^{I}\left(z_{\iota}^{I}(t)\right), \quad f_{j}^{I}\left(z_{j}^{I}(t)\right), \quad h_{j}^{I}\left(z_{j}^{I}(t-\right.$ $\left.\tau_{j}(t)\right)$ are the imaginary parts of $z_{\iota}(t), \quad b_{\iota j}\left(z_{\iota}(t)\right), \quad d_{\iota j}\left(z_{\iota}(t)\right), f_{j}\left(z_{j}(t)\right), h_{j}\left(z_{j}\left(t-\tau_{j}(t)\right)\right)$, respectively.

In accordance with the voltage-current characteristic of memristor, one has

$$
\begin{aligned}
b_{\iota j}^{R}\left(z_{\iota}^{R}(t)\right) & = \begin{cases}\hat{b}_{\iota j}^{R}, & \left|z_{\iota}^{R}(t)\right| \leqslant \Gamma_{\iota}, \\
\breve{b}_{\iota j}^{R}, & \left|z_{\iota}^{R}(t)\right|>\Gamma_{\iota},\end{cases} \\
b_{\iota j}^{I}\left(z_{\iota}^{I}(t)\right) & = \begin{cases}\hat{b}_{\iota j}^{I}, & \left|z_{\iota}^{I}(t)\right| \leqslant \Gamma_{\iota}, \\
\check{b}_{\iota j}^{I}, & \left|z_{\iota}^{I}(t)\right|>\Gamma_{\iota},\end{cases} \\
d_{\iota j}^{R}\left(z_{\iota}^{R}(t)\right) & = \begin{cases}\hat{d}_{\iota j}^{R}, & \left|z_{\iota}^{R}(t)\right| \leqslant \Gamma_{\iota}, \\
\check{d}_{\iota j}^{R}, & \left|z_{\iota}^{R}(t)\right|>\Gamma_{\iota},\end{cases} \\
d_{\iota j}^{I}\left(z_{\iota}^{I}(t)\right) & = \begin{cases}\hat{d}_{\iota j}^{I}, & \left|z_{\iota}^{I}(t)\right| \leqslant \Gamma_{\iota}, \\
\check{d}_{\iota j}^{I}, & \left|z_{\iota}^{I}(t)\right|>\Gamma_{\iota},\end{cases}
\end{aligned}
$$

where $\iota, j \in\{1,2, \cdots, n\} ; \hat{b}_{\iota j}^{R}, \hat{b}_{\iota j}^{I}, \check{b}_{\iota j}^{R}, \check{b}_{\iota j}^{I}, \hat{d}_{\iota j}^{R}, \hat{d}_{\iota j}^{I}, \breve{d}_{\iota j}^{R}, \check{d}_{\iota j}^{I}$ are all constants. $\Gamma_{\iota}>0$ represents the threshold level.
Let $\tilde{b}_{\iota j}^{R}=\max \left\{\left|\hat{b}_{\iota j}^{R}\right|,\left|\check{b}_{\iota j}^{R}\right|\right\}, \quad \tilde{b}_{\iota j}^{I}=\max \left\{\left|\hat{b}_{\iota j}^{I}\right|,\left|\check{b}_{\iota j}^{I}\right|\right\}$, $\tilde{d}_{\iota j}^{R}=\max \left\{\left|\hat{d}_{\iota j}^{R}\right|,\left|\breve{d}_{\iota j}^{R}\right|\right\}, \quad \tilde{d}_{\iota j}^{I}=\max \left\{\left|\hat{d}_{\iota j}^{I}\right|,\left|\breve{d}_{\iota j}^{I}\right|\right\}$, $\bar{b}_{\iota j}^{R}=\left|\hat{b}_{\iota j}^{R}-\check{b}_{\iota j}^{R}\right|, \bar{b}_{\iota j}^{I}=\left|\hat{b}_{\iota j}^{I}-\check{b}_{\iota j}^{I}\right|, \bar{d}_{\iota j}^{R}=\left|\hat{d}_{\iota j}^{R}-\check{d}_{\iota j}^{R}\right|$, $\bar{d}_{\iota j}^{I}=\left|\hat{d}_{\iota j}^{I}-\tilde{d}_{\iota j}^{I}\right|, \bar{B}^{R}=\left(\bar{b}_{\iota j}^{R}\right)_{n \times n}, \bar{B}^{I}=\left(\bar{b}_{\iota j}^{I}\right)_{n \times n}$, $\tilde{B}^{R}=\operatorname{diag}\left(\sum_{j=1}^{n}\left(\tilde{b}_{1 j}^{R}\right)^{2}, \sum_{j=1}^{n}\left(\tilde{b}_{2 j}^{R}\right)^{2}, \cdots, \sum_{j=1}^{n}\left(b_{n j}^{R}\right)^{2}\right)$, $\tilde{B}^{I}=\operatorname{diag}\left(\sum_{j=1}^{n}\left(\tilde{b}_{1 j}^{I}\right)^{2}, \sum_{j=1}^{n}\left(\tilde{b}_{2 j}^{I}\right)^{2}, \cdots, \sum_{j=1}^{n}\left(\tilde{b}_{n j}^{I}\right)^{2}\right)$, $\bar{D}^{R}=\left(\bar{d}_{\iota j}^{R}\right)_{n \times n}, \quad \bar{D}^{I}=\left(\bar{d}_{\iota j}^{I}\right)_{n \times n}, \quad \tilde{D}^{R}=$ $\operatorname{diag}\left(\sum_{j=1}^{n}\left(\tilde{d}_{1 j}^{R}\right)^{2}, \sum_{j=1}^{n}\left(\tilde{d}_{2 j}^{R}\right)^{2}, \cdots, \sum_{j=1}^{n}\left(\tilde{d}_{n j}^{R}\right)^{2}\right), \quad \tilde{D}^{I}=$ $\operatorname{diag}\left(\sum_{j=1}^{n}\left(\tilde{d}_{1 j}^{I}\right)^{2}, \sum_{j=1}^{n}\left(\tilde{d}_{2 j}^{I}\right)^{2}, \cdots, \sum_{j=1}^{n}\left(\tilde{d}_{n j}^{I}\right)^{2}\right)$.

In this section, we consider the following CCVMNNs consisting of $N$ CVMNNs (1):

$$
\begin{aligned}
\dot{Z}_{s}(t)= & -A Z_{s}(t)+B\left(Z_{s}(t)\right) h\left(\overline{Z_{s}(t)}\right)+D\left(Z_{s}(t)\right) f\left(Z_{s}(t)\right)+u_{s}(t) \\
& +g \sum_{\kappa=1}^{N} G_{s \kappa} M Z_{\kappa}(t)+x_{s}(t), s=1,2, \cdots, N, \quad \text { (2) }
\end{aligned}
$$

where $Z_{s}(t)=\left(Z_{s 1}(t), Z_{s 2}(t), \cdots, Z_{s n}(t)\right) \in \mathbb{C}^{n}$ represents the complex-valued state variable of the $s$ th node. $0<A=\operatorname{diag}\left(a_{1}, a_{2}, \cdots, a_{n}\right) \in \mathbb{R}^{n \times n}$. $\overline{Z_{s}(t)}=\left(Z_{s 1}\left(t-\tau_{1}(t)\right), Z_{s 2}\left(t-\tau_{2}(t)\right), \cdots, Z_{s n}(t-\right.$ $\left.\left.\tau_{n}(t)\right)\right)^{T} \in \mathbb{C}^{n} \cdot h\left(\overline{Z_{s}(t)}\right)=\left(h_{1}\left(Z_{s 1}\left(t-\tau_{1}(t)\right)\right), h_{2}\left(Z_{s 2}(t-\right.\right.$ $\left.\left.\left.\tau_{2}(t)\right)\right), \cdots, h_{n}\left(Z_{s n}\left(t-\tau_{n}(t)\right)\right)\right)^{T} \in \mathbb{C}^{n} \cdot f\left(Z_{s}(t)\right)=$ $\left(f_{1}\left(Z_{s 1}(t)\right), f_{2}\left(Z_{s 2}(t)\right), \cdots, f_{n}\left(Z_{s n}(t)\right)\right)^{T} \in \mathbb{C}^{n} ; M \in$ $\mathbb{R}^{n \times n}$ symbols the inner coupling matrix. $B\left(Z_{s}(t)\right)=$ $\left(b_{\iota j}\left(Z_{s \iota}(t)\right)\right)_{n \times n} \in \mathbb{C}^{n \times n}, D\left(Z_{s}(t)\right)=\left(d_{\iota j}\left(Z_{s \iota}(t)\right)\right)_{n \times n} \in$ $\mathbb{C}^{n \times n}$, where $\iota, j=1,2, \cdots, n . u_{s}(t)=u_{s}^{R}(t)+i u_{s}^{I}(t)=$ $\left(u_{s 1}(t), u_{s 2}(t), \cdots, u_{s n}(t)\right)^{T} \in \mathbb{C}^{n}$ is the controller to be designed for obtaining a certain control objective. $x_{s}(t)=$ $x_{s}^{R}(t)+i x_{s}^{I}(t)=\left(x_{s 1}(t), x_{s 2}(t), \cdots, x_{s n}(t)\right)^{T} \in \mathbb{C}^{n}$ denotes the external input of the network. $g>0$ is the overall coupling strength. $G=\left(G_{S \kappa}\right)_{N \times N}$ stands for coupling weight between nodes, where $G_{s \kappa}=G_{\kappa s}>0$ if and only if there exists a connection between node s and node $\kappa$; if not, $G_{s \kappa}=G_{\kappa s}=0(s \neq \kappa)$; and

$$
G_{s s}=-\sum_{\substack{\kappa=1 \\ \kappa \neq s}}^{N} G_{s \kappa}, s=1,2, \cdots, N .
$$

Then, the network (2) can be separated into real and imaginary parts as follows:

$$
\begin{aligned}
\dot{Z}_{s}^{R}(t)= & -A Z_{s}^{R}(t)+B^{R}\left(Z_{s}^{R}(t)\right) h^{R}\left(\overline{Z_{s}^{R}(t)}\right)+u_{s}^{R}(t)+x_{s}^{R}(t) \\
& -B^{I}\left(Z_{s}^{I}(t)\right) h^{I}\left(\overline{Z_{s}^{I}(t)}\right)+D^{R}\left(Z_{s}^{R}(t)\right) f^{R}\left(Z_{s}^{R}(t)\right) \\
& -D^{I}\left(Z_{s}^{I}(t)\right) f^{I}\left(Z_{s}^{I}(t)\right)+g \sum_{\kappa=1}^{N} G_{s \kappa} M Z_{\kappa}^{R}(t), \\
\dot{Z}_{s}^{I}(t)= & -A Z_{s}^{I}(t)+B^{R}\left(Z_{s}^{R}(t)\right) h^{I}\left(\overline{Z_{s}^{I}(t)}\right)+u_{s}^{I}(t)+x_{s}^{I}(t) \\
& +B^{I}\left(Z_{s}^{I}(t)\right) h^{R}\left(\overline{Z_{s}^{R}(t)}\right)+D^{R}\left(Z_{s}^{R}(t)\right) f^{I}\left(Z_{s}^{I}(t)\right) \\
& +D^{I}\left(Z_{s}^{I}(t)\right) f^{R}\left(Z_{s}^{R}(t)\right)+g \sum_{\kappa=1}^{N} G_{s \kappa} M Z_{\kappa}^{I}(t),
\end{aligned}
$$

where $\quad h^{R}\left(\overline{Z_{s}^{R}(t)}\right) \quad=\quad\left(h_{1}^{R}\left(Z_{s 1}^{R}(t \quad-\right.\right.$ $\left.\left.\left.\tau_{1}(t)\right)\right), h_{2}^{R}\left(Z_{s 2}^{R}\left(t-\tau_{2}(t)\right)\right), \cdots, h_{n}^{R}\left(Z_{s n}^{R}\left(t-\tau_{n}(t)\right)\right)\right)^{T}$, $h^{I}\left(\overline{Z_{s}^{I}(t)}\right)=\left(h_{1}^{I}\left(Z_{s 1}^{I}\left(t-\tau_{1}(t)\right)\right), h_{2}^{I}\left(Z_{s 2}^{I}(t\right.\right.$ 
$\left.\left.\left.\tau_{2}(t)\right)\right), \cdots, h_{n}^{I}\left(Z_{s n}^{I}\left(t \quad-\quad \tau_{n}(t)\right)\right)\right)^{T}, \quad f^{R}\left(Z_{s}^{R}(t)\right)=$ $\left(f_{1}^{R}\left(Z_{s 1}^{R}(t)\right), f_{2}^{R}\left(Z_{s 2}^{R}(t)\right), \cdots, f_{n}^{R}\left(Z_{s n}^{R}(t)\right)\right)^{T}, \quad f^{I}\left(Z_{s}^{I}(t)\right)=$ $\left(f_{1}^{I}\left(Z_{s 1}^{I}(t)\right), f_{2}^{I}\left(Z_{s 2}^{I}(t)\right), \cdots, f_{n}^{I}\left(Z_{s n}^{I}(t)\right)\right)^{T}, \quad Z_{s}^{R}(t)=$ $\left(Z_{s 1}^{R}(t), Z_{s 2}^{R}(t), \cdots, Z_{s n}^{R}(t)\right)^{T}, \quad B^{R}(\cdot) \quad=$ $\left(b_{\iota j}^{R}(\cdot)\right)_{n \times n}, Z_{s}^{I}(t)=\left(Z_{s 1}^{I}(t), Z_{s 2}^{I}(t), \cdots, Z_{s n}^{I}(t)\right)^{T}, D^{R}(\cdot)=$ $\left(d_{\iota j}^{R}(\cdot)\right)_{n \times n}, u_{s}^{R}(t)=\left(u_{s 1}^{R}(t), u_{s 2}^{R}(t), \cdots, u_{s n}^{R}(t)\right)^{T}, B^{I}(\cdot)=$ $\left(b_{\iota j}^{I}(\cdot)\right)_{n \times n}, u_{s}^{I}(t)=\left(u_{s 1}^{I}(t), u_{s 2}^{I}(t), \cdots, u_{s n}^{I}(t)\right)^{T}, D^{I}(\cdot)=$ $\left(d_{\iota j}^{I}(\cdot)\right)_{n \times n}, x_{s}^{R}(t)=\left(x_{s 1}^{R}(t), x_{s 2}^{R}(t), \cdots, x_{s n}^{R}(t)\right)^{T}, x_{s}^{I}(t)=$ $\left(x_{s 1}^{I}(t), x_{s 2}^{I}(t), \cdots, x_{s n}^{I}(t)\right)^{T}$.

Assumption 1. For any $\alpha_{1}, \alpha_{2} \in \mathbb{R}$, the real part $f_{s}^{R}(\cdot)$ and the imaginary part $f_{s}^{I}(\cdot)$ of function $f_{s}(\cdot)$ and the real part $h_{s}^{R}(\cdot)$ and the imaginary part $h_{s}^{I}(\cdot)$ of function $h_{s}(\cdot)$ satisfy

$$
\begin{aligned}
& \left|f_{s}^{R}(\cdot)\right| \leqslant F_{s}^{R},\left|f_{s}^{I}(\cdot)\right| \leqslant F_{s}^{I} \\
& \left|h_{s}^{R}(\cdot)\right| \leqslant H_{s}^{R},\left|h_{s}^{I}(\cdot)\right| \leqslant H_{s}^{I} \\
& \left|f_{s}^{R}\left(\alpha_{1}\right)-f_{s}^{R}\left(\alpha_{2}\right)\right| \leqslant l_{s}^{R}\left|\alpha_{1}-\alpha_{2}\right|, \\
& \left|f_{s}^{I}\left(\alpha_{1}\right)-f_{s}^{I}\left(\alpha_{2}\right)\right| \leqslant l_{s}^{I}\left|\alpha_{1}-\alpha_{2}\right| \\
& \left|h_{s}^{R}\left(\alpha_{1}\right)-h_{s}^{R}\left(\alpha_{2}\right)\right| \leqslant \eta_{s}^{R}\left|\alpha_{1}-\alpha_{2}\right|, \\
& \left|h_{s}^{I}\left(\alpha_{1}\right)-h_{s}^{I}\left(\alpha_{2}\right)\right| \leqslant \eta_{s}^{I}\left|\alpha_{1}-\alpha_{2}\right|
\end{aligned}
$$

where $F_{s}^{R}, F_{s}^{I}, H_{s}^{R}, H_{s}^{I}, l_{s}^{R}, l_{s}^{I}, \eta_{s}^{R}, \eta_{s}^{I}$ are positive constants.

Suppose $Z_{0}(t)=\left(Z_{01}(t), Z_{02}(t), \cdots, Z_{0 n}(t)\right)^{T} \in \mathbb{C}^{n}$ is an arbitrary solution of the network (2), then

$\dot{Z}_{0}(t)=-A Z_{0}(t)+B\left(Z_{0}(t)\right) h\left(Z_{0}(t)\right)+D\left(Z_{0}(t)\right) f\left(Z_{0}(t)\right)$,

where $Z_{0}(t)=Z_{0}^{R}(t)+i Z_{0}^{I}(t)$. Then, Eq. (5) can be separated into real and imaginary parts as follows:

$$
\begin{aligned}
& \dot{Z}_{0}^{R}(t)=-A Z_{0}^{R}(t)+B^{R}\left(Z_{0}^{R}(t)\right) h^{R}\left(Z_{0}^{R}(t)\right)-B^{I}\left(Z_{0}^{I}(t)\right) h^{I}\left(Z_{0}^{I}(t)\right) \\
&+D^{R}\left(Z_{0}^{R}(t)\right) f^{R}\left(Z_{0}^{R}(t)\right)-D^{I}\left(Z_{0}^{I}(t)\right) f^{I}\left(Z_{0}^{I}(t)\right) \\
& \dot{Z}_{0}^{I}(t)=- A Z_{0}^{I}(t)+B^{R}\left(Z_{0}^{R}(t)\right) h^{I}\left(Z_{0}^{I}(t)\right)+B^{I}\left(Z_{0}^{I}(t)\right) h^{R}\left(Z_{0}^{R}(t)\right) \\
&+D^{R}\left(Z_{0}^{R}(t)\right) f^{I}\left(Z_{0}^{I}(t)\right)+D^{I}\left(Z_{0}^{I}(t)\right) f^{R}\left(Z_{0}^{R}(t)\right) . \\
& \text { Let } e_{s}(t)= Z_{s}(t)-Z_{0}(t), \text { then } \\
& \dot{e}_{s}(t)=-A e_{s}(t)+B\left(Z_{s}(t)\right) h\left(\overline{Z_{s}(t)}\right)-B\left(Z_{0}(t)\right) h\left(Z_{0}(t)\right) \\
& \quad+D\left(Z_{s}(t)\right) f\left(Z_{s}(t)\right)+u_{s}(t)-D\left(Z_{0}(t)\right) f\left(Z_{0}(t)\right) \\
& \\
&+g \sum_{\kappa=1}^{N} G_{s \kappa} M e_{\kappa}(t)+x_{s}(t), s=1,2, \cdots, N
\end{aligned}
$$

where $e_{s}(t)=\left(e_{s 1}(t), e_{s 2}(t), \cdots, e_{s n}(t)\right)^{T}$.

By separating (6) into real and imaginary parts, one has

$$
\begin{aligned}
\dot{e}_{s}^{R}(t)= & -A e_{s}^{R}(t)+D^{R}\left(Z_{s}^{R}(t)\right) P^{R}\left(e_{s}^{R}(t)\right)+x_{s}^{R}(t)+u_{s}^{R}(t) \\
& -D^{I}\left(Z_{s}^{I}(t)\right) P^{I}\left(e_{s}^{I}(t)\right)+B^{R}\left(Z_{s}^{R}(t)\right) Q^{R}\left(\overline{e_{s}^{R}(t)}\right) \\
& -B^{I}\left(Z_{s}^{I}(t)\right) Q^{I}\left(\overline{e_{s}^{I}(t)}\right)+g \sum_{\kappa=1}^{N} G_{s \kappa} M e_{\kappa}^{R}(t) \\
& +\left(D^{R}\left(Z_{s}^{R}(t)\right)-D^{R}\left(Z_{0}^{R}(t)\right)\right) f^{R}\left(Z_{0}^{R}(t)\right) \\
& +\left(B^{R}\left(Z_{s}^{R}(t)\right)-B^{R}\left(Z_{0}^{R}(t)\right)\right) h^{R}\left(Z_{0}^{R}(t)\right) \\
& -\left(D^{I}\left(Z_{s}^{I}(t)\right)-D^{I}\left(Z_{0}^{I}(t)\right)\right) f^{I}\left(Z_{0}^{I}(t)\right) \\
& -\left(B^{I}\left(Z_{s}^{I}(t)\right)-B^{I}\left(Z_{0}^{I}(t)\right)\right) h^{I}\left(Z_{0}^{I}(t)\right),
\end{aligned}
$$

$$
\begin{aligned}
\dot{e}_{s}^{I}(t)= & -A e_{s}^{I}(t)+D^{R}\left(Z_{s}^{R}(t)\right) P^{I}\left(e_{s}^{I}(t)\right)+x_{s}^{I}(t)+u_{s}^{I}(t) \\
& +D^{I}\left(Z_{s}^{I}(t)\right) P^{R}\left(e_{s}^{R}(t)\right)+B^{R}\left(Y_{s}^{R}(t)\right) Q^{I}\left(\overline{e_{s}^{I}(t)}\right) \\
& +B^{I}\left(Z_{s}^{I}(t)\right) Q^{R}\left(\overline{e_{s}^{R}(t)}\right)+g \sum_{\kappa=1}^{N} G_{s \kappa} M e_{\kappa}^{I}(t) \\
& +\left(D^{R}\left(Z_{s}^{R}(t)\right)-D^{R}\left(Z_{0}^{R}(t)\right)\right) f^{I}\left(Z_{0}^{I}(t)\right) \\
& +\left(D^{I}\left(Z_{s}^{I}(t)\right)-D^{I}\left(Z_{0}^{I}(t)\right)\right) f^{R}\left(Z_{0}^{R}(t)\right) \\
& +\left(B^{R}\left(Z_{s}^{R}(t)\right)-B^{R}\left(Z_{0}^{R}(t)\right)\right) h^{I}\left(Z_{0}^{I}(t)\right) \\
& +\left(B^{I}\left(Z_{s}^{I}(t)\right)-B^{I}\left(Z_{0}^{I}(\varrho, t)\right)\right) h^{R}\left(Z_{0}^{R}(t)\right),
\end{aligned}
$$

where $e_{s}^{R}(t)=\left(e_{s 1}^{R}(t), e_{s 2}^{R}(t), \cdots, e_{s n}^{R}(t)\right)^{T}, \quad e_{s}^{I}(t)=$ $\left(e_{s 1}^{I}(t), e_{s 2}^{I}(t), \cdots, e_{s n}^{I}(t)\right)^{T}, \quad \overline{e_{s}^{R}(t)} \stackrel{s n}{=} \quad \frac{\left(e_{s 1}^{R}(t-\right.}{e_{s}^{I}(t)}$ $\left.\left.\tau_{1}(t)\right), e_{s 2}^{R}\left(t-\tau_{2}(t)\right), \cdots, e_{s n}^{R}\left(t-\tau_{n}(t)\right)\right)^{T}, \quad \overline{e_{s}^{I}(t)}=$ $\left(e_{s 1}^{I}\left(t-\tau_{1}(t)\right), e_{s 2}^{I}\left(t-\tau_{2}(t)\right), \cdots, e_{s n}^{I}\left(t-\tau_{n}(t)\right)\right)^{T}$,

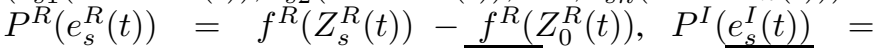
$f^{I}\left(Z_{s}^{I}(t)\right)-f^{I}\left(Z_{0}^{I}(t)\right), \quad Q^{R}\left(\frac{e_{s}^{R}(t)}{}\right)=h^{R}\left(\overline{Z_{s}^{R}(t)}\right)-$ $h^{R}\left(Z_{0}^{R}(t)\right)$ and $Q^{I}\left(\overline{e_{s}^{I}(t)}\right)=h^{I}\left(\overline{Z_{s}^{I}(t)}\right)-h^{I}\left(Z_{0}^{I}(t)\right)$.

Definition III.1. For any $t_{2}, t_{1} \in \mathbb{R}^{+}$and $t_{2} \geqslant t_{1}$, if there exists a constant $\rho>0$ satisfy

$$
\begin{aligned}
& \int_{t_{1}}^{t_{2}}\left[\left(y^{R}(t)\right)^{T} x^{R}(t)+\left(y^{I}(t)\right)^{T} x^{I}(t)\right] d t \geqslant \\
& V\left(t_{2}\right)-V\left(t_{1}\right)-\rho \int_{t_{1}}^{t_{2}}\left[\left(x^{R}(t)\right)^{T} x^{R}(t)+\left(x^{I}(t)\right)^{T} x^{I}(t)\right] d t,
\end{aligned}
$$

where $V(t): \mathbb{R}^{+} \rightarrow \mathbb{R}^{+}$is the storage function, then the network (6) is called passive.

Definition III.2. The network (2) is synchronized if

$$
\lim _{t \rightarrow \infty}\left\|Z_{s}(t)-Z_{0}(t)\right\|=0, \quad s=1,2, \cdots, N,
$$

under the condition $x_{s}(t)=0, s=1,2, \cdots, N$.

\section{B. Passivity control}

The following state feedback controller is designed for the network (2):

$$
\left\{\begin{aligned}
u_{s}^{R}(t)= & -\Upsilon^{R} e_{s}^{R}(t)-\operatorname{sign}\left(e_{s}^{R}(t)\right)\left(\bar{D}^{R} \bar{F}^{R}+\bar{D}^{I} \bar{F}^{I}\right. \\
& \left.+\bar{B}^{R} \bar{H}^{R}+\bar{B}^{I} \bar{H}^{I}\right), \\
u_{s}^{I}(t)= & -\Upsilon^{I} e_{s}^{I}(t)-\operatorname{sign}\left(e_{s}^{I}(t)\right)\left(\bar{D}^{R} \bar{F}^{I}+\bar{D}^{I} \bar{F}^{R}\right. \\
& \left.+\bar{B}^{R} \bar{H}^{I}+\bar{B}^{I} \bar{H}^{R}\right),
\end{aligned}\right.
$$

where $s=1,2, \cdots, N, \Upsilon^{R}=\operatorname{diag}\left(v_{1}^{R}, v_{2}^{R}, \cdots, v_{n}^{R}\right) \in$ $\mathbb{R}^{n \times n}$ and $\Upsilon^{I}=\operatorname{diag}\left(v_{1}^{I}, v_{2}^{I}, \cdots, v_{n}^{I}\right) \in \mathbb{R}^{n \times n}$ are the positive definite controller gain matrices. $\mathbb{R} \ni v_{\iota}^{R}>0$ and $\mathbb{R} \ni v_{\iota}^{I}>0 . \bar{F}^{R}=\left(F_{1}^{R}, F_{2}^{R}, \cdots, F_{n}^{R}\right)^{T}$, $\bar{F}^{I}=\left(F_{1}^{I}, F_{2}^{I}, \cdots, F_{n}^{I}\right)^{T}, \bar{H}^{R}=\left(H_{1}^{R}, H_{2}^{R}, \cdots, H_{n}^{R}\right)^{T}$, and $\bar{H}^{I}=\left(H_{1}^{I}, H_{2}^{I}, \cdots, H_{n}^{I}\right)^{T} \cdot \operatorname{sign}\left(e_{s}^{R}(t)\right)=$ $\operatorname{diag}\left(\operatorname{sign}\left(e_{s 1}^{R}(t)\right), \operatorname{sign}\left(e_{s 2}^{R}(t)\right), \cdots, \operatorname{sign}\left(e_{s n}^{R}(t)\right)\right) \quad$ and $\operatorname{sign}\left(e_{s}^{I}(t)\right)=\operatorname{diag}\left(\operatorname{sign}\left(e_{s 1}^{I}(t)\right), \operatorname{sign}\left(e_{s 2}^{I}(t)\right), \cdots, \operatorname{sign}\left(e_{s n}^{I}(t)\right)\right)$.

The output vector $y_{s}(t) \in \mathbb{C}^{n}$ of the system (6) is described as follows:

$$
y_{s}(t)=W_{1} e_{s}(t)+W_{2} x_{s}(t)
$$

where $W_{1} \in \mathbb{R}^{n \times n}$ and $W_{2} \in \mathbb{R}^{n \times n}$. 
For convenience, we denote

$$
\begin{aligned}
& e^{R}(t)=\left(\left(e_{1}^{R}(t)\right)^{T},\left(e_{2}^{R}(t)\right)^{T}, \cdots,\left(e_{N}^{R}(t)\right)^{T}\right)^{T}, \\
& e^{I}(t)=\left(\left(e_{1}^{I}(t)\right)^{T},\left(e_{2}^{I}(t)\right)^{T}, \cdots,\left(e_{N}^{I}(t)\right)^{T}\right)^{T}, \\
& \overline{e^{R}(t)}=\left(\left(\overline{e_{1}^{R}(t)}\right)^{T},\left(\overline{e_{2}^{R}(t)}\right)^{T}, \cdots,\left(\overline{e_{N}^{R}(t)}\right)^{T}\right)^{T}, \\
& \overline{e^{I}(t)}\left.=\left(\overline{\left(e_{1}^{I}(t)\right.}\right)^{T},\left(\overline{e_{2}^{I}(t)}\right)^{T}, \cdots,\left(\overline{e_{N}^{I}(t)}\right)^{T}\right)^{T}, \\
& L^{R}=\operatorname{diag}\left(\left(l_{1}^{R}\right)^{2},\left(l_{2}^{R}\right)^{2}, \cdots,\left(l_{n}^{R}\right)^{2}\right), \\
& L^{I}=\operatorname{diag}\left(\left(l_{1}^{I}\right)^{2},\left(l_{2}^{I}\right)^{2}, \cdots,\left(l_{n}^{I}\right)^{2}\right), \\
& \zeta^{R}=\operatorname{diag}\left(\left(\eta_{1}^{R}\right)^{2},\left(\eta_{2}^{R}\right)^{2}, \cdots,\left(\eta_{n}^{R}\right)^{2}\right), \\
& \zeta^{I}=\operatorname{diag}\left(\left(\eta_{1}^{I}\right)^{2},\left(\eta_{2}^{I}\right)^{2}, \cdots,\left(\eta_{n}^{I}\right)^{2}\right), \\
& x(t)=\left(x_{1}^{H}(t), x_{2}^{H}(t), \cdots, x_{N}^{H}(t)\right)^{H}, \\
& y(t)=\left(y_{1}^{H}(t), y_{2}^{H}(t), \cdots, y_{N}^{H}(t)\right)^{H}, \\
& \Gamma=\operatorname{diag}\left(\frac{1}{1-\gamma_{1}}, \frac{1}{1-\gamma_{2}}, \cdots, \frac{1}{1-\gamma_{n}}\right) .
\end{aligned}
$$

Theorem III.1. If there exists a constant $\rho>0$ such that

$$
\left(\begin{array}{ll}
\Psi_{1}^{R} & \Xi^{R} \\
\left(\Xi^{R}\right)^{T} & \Psi_{2}^{R}
\end{array}\right) \leqslant 0 \text { and }\left(\begin{array}{ll}
\Psi_{1}^{I} & \Xi^{I} \\
\left(\Xi^{I}\right)^{T} & \Psi_{2}^{I}
\end{array}\right) \leqslant 0,
$$

where $\Psi_{1}^{R}=I_{N} \otimes\left(-2 A+\tilde{D}^{R}+2 L^{R}+\tilde{D}^{I}+\tilde{B}^{R}+\tilde{B}^{I}-\Upsilon^{R}+\right.$ $\left.2 \zeta^{R} \Gamma\right)+g G \otimes\left(M+M^{T}\right), \Xi^{R}=\Xi^{I}=I_{N} \otimes\left(I_{n}-\frac{1}{2} W_{1}^{T}\right)$, $\Psi_{1}^{I}=I_{N} \otimes\left(-2 A+2 L^{I}+\tilde{D}^{R}+\tilde{D}^{I}+\tilde{B}^{I}+\tilde{B}^{R}-\Upsilon^{I}+2 \zeta^{I} \Gamma\right)+$ $g G \otimes\left(M+M^{T}\right), \Psi_{2}^{R}=\Psi_{2}^{I}=I_{N} \otimes\left(-\frac{1}{2}\left(W_{2}^{T}+W_{2}\right)-\rho I_{n}\right)$, then the network (6) is said to be passive under the controller (7).

Proof. We construct a Lyapunov functional as follows:

$$
\begin{aligned}
V(t)= & \sum_{s=1}^{N}\left(e_{s}^{R}(t)\right)^{T} e_{s}^{R}(t)+2 \sum_{s=1}^{N} \sum_{j=1}^{n} \int_{t-\tau_{j}(t)}^{t} \frac{\left(\eta_{j}^{R} e_{s j}^{R}(\delta)\right)^{2}}{1-\gamma_{j}} d \delta \\
& +2 \sum_{s=1}^{N} \sum_{j=1}^{n} \int_{t-\tau_{j}(t)}^{t} \frac{\left(\eta_{j}^{I} e_{s j}^{I}(\delta)\right)^{2}}{1-\gamma_{j}} d \delta+\sum_{s=1}^{N}\left(e_{s}^{I}(t)\right)^{T} e_{s}^{I}(t) .
\end{aligned}
$$

Then,

$$
\begin{aligned}
\dot{V}(t) \leqslant & 2 \sum_{s=1}^{N}\left(e_{s}^{R}(t)\right)^{T}\left(-A e_{s}^{R}(t)+D^{R}\left(Z_{s}^{R}(t)\right) P^{R}\left(e_{s}^{R}(t)\right)+x_{s}^{R}(t)\right. \\
& -D^{I}\left(Z_{s}^{I}(t)\right) P^{I}\left(e_{s}^{I}(t)\right)+B^{R}\left(Z_{s}^{R}(t)\right) Q^{R}\left(\overline{e_{s}^{R}(t)}\right)-\Upsilon^{R} e_{s}^{R}(t) \\
& -B^{I}\left(Z_{s}^{I}(t)\right) Q^{I}\left(\overline{e_{s}^{I}(t)}\right)+g \sum_{\kappa=1}^{N} G_{s \kappa} M e_{\kappa}^{R}(t)+\left(D^{R}\left(Z_{s}^{R}(t)\right)\right. \\
& \left.-D^{R}\left(Z_{0}^{R}(t)\right)\right) f^{R}\left(Z_{0}^{R}(t)\right)-\operatorname{sign}\left(e_{s}^{R}(t)\right)\left(\bar{D}^{R} \bar{F}^{R}+\bar{D}^{I} \bar{F}^{I}\right. \\
& \left.+\bar{B}^{R} \bar{H}^{R}+\bar{B}^{I} \bar{H}^{I}\right)-\left(D^{I}\left(Z_{s}^{I}(t)\right)-D^{I}\left(Z_{0}^{I}(t)\right)\right) f^{I}\left(Z_{0}^{I}(t)\right) \\
& +\left(B^{R}\left(Z_{s}^{R}(t)\right)-B^{R}\left(Z_{0}^{R}(t)\right)\right) h^{R}\left(Z_{0}^{R}(t)\right)-\left(B^{I}\left(Z_{s}^{I}(t)\right)\right. \\
& \left.\left.-B^{I}\left(Z_{0}^{I}(t)\right)\right) h^{I}\left(Z_{0}^{I}(t)\right)\right)+2 \sum_{s=1}^{N}\left(e_{s}^{I}(t)\right)^{T}\left(-A e_{s}^{I}(t)+x_{s}^{I}(t)\right.
\end{aligned}
$$$$
+D^{R}\left(Z_{s}^{R}(t)\right) P^{I}\left(e_{s}^{I}(t)\right)+D^{I}\left(Z_{s}^{I}(t)\right) P^{R}\left(e_{s}^{R}(t)\right)-\Upsilon^{I} e_{s}^{I}(t)
$$$$
+B^{R}\left(Z_{s}^{R}(t)\right) Q^{I}\left(\overline{e_{s}^{I}(t)}\right)+B^{I}\left(Z_{s}^{I}(t)\right) Q^{R}\left(\overline{e_{s}^{R}(t)}\right)+\left(D^{R}\left(Z_{s}^{R}(t)\right)\right. \text { In addition, }
$$$$
\left.-D^{R}\left(Z_{0}^{R}(t)\right)\right) f^{I}\left(Z_{0}^{I}(t)\right)+g \sum_{\kappa=1}^{N} G_{s \kappa} M e_{\kappa}^{I}(t)+\left(D^{I}\left(Z_{s}^{I}(t)\right)\right.
$$$$
\text { - } \left.D^{I}\left(Z_{0}^{I}(t)\right)\right) f^{R}\left(Z_{0}^{R}(t)\right)+\left(B^{R}\left(Z_{s}^{R}(t)\right)-B^{R}\left(Z_{0}^{R}(t)\right)\right)
$$$$
\times h^{I}\left(Z_{0}^{I}(t)\right)-\operatorname{sign}\left(e_{s}^{I}(t)\right)\left(\bar{D}^{R} \bar{F}^{I}+\bar{D}^{I} \bar{F}^{R}+\bar{B}^{R} \bar{H}^{I}+\bar{B}^{I} \bar{H}^{R}\right)
$$$$
\left.+\left(B^{I}\left(Z_{s}^{I}(t)\right)-B^{I}\left(Z_{0}^{I}(t)\right)\right) h^{R}\left(Z_{0}^{R}(t)\right)\right)+2\left(e^{R}(t)\right)^{T}\left(I_{N}\right.
$$$$
\left.\otimes\left(\zeta^{R} \Gamma\right)\right) e^{R}(t)-2\left(\overline{e^{R}(t)}\right)^{T}\left(I_{N} \otimes \zeta^{R}\right) \overline{e^{R}(t)}+2\left(e^{I}(t)\right)^{T}
$$$$
\times\left(I_{N} \otimes\left(\zeta^{I} \Gamma\right)\right) e^{I}(t)-2\left(\overline{e^{I}(t)}\right)^{T}\left(I_{N} \otimes \zeta^{I}\right) \overline{e^{I}(t)} .
$$

From Assumption 1, one has

$$
\begin{aligned}
& 2 \sum_{s=1}^{N}\left(e_{s}^{R}(t)\right)^{T} D^{R}\left(Z_{s}^{R}(t)\right) P^{R}\left(e_{s}^{R}(t)\right) \\
\leqslant & 2 \sum_{s=1}^{N} \sum_{\iota=1}^{n} \sum_{j=1}^{n}\left|e_{s \iota}^{R}(t)\right| \tilde{d}_{\iota j}^{R}\left|f_{j}^{R}\left(Z_{s j}^{R}(t)\right)-f_{j}^{R}\left(Z_{0 j}^{R}(t)\right)\right| \\
\leqslant & \sum_{s=1}^{N} \sum_{\iota=1}^{n} \sum_{j=1}^{n}\left(e_{s \iota}^{R}(t)\right)^{2}\left(\tilde{d}_{\iota j}^{R}\right)^{2}+\sum_{s=1}^{N} \sum_{j=1}^{n}\left(l_{j}^{R}\right)^{2}\left(e_{s j}^{R}(t)\right)^{2} \\
= & \left(e^{R}(t)\right)^{T}\left(I_{N} \otimes \tilde{D}^{R}\right) e^{R}(t)+\left(e^{R}(t)\right)^{T}\left(I_{N} \otimes L^{R}\right) e^{R}(t) .
\end{aligned}
$$

Similarly,

$$
-2 \sum_{s=1}^{N}\left(e_{s}^{R}(t)\right)^{T} D^{I}\left(Z_{s}^{I}(t)\right) P^{I}\left(e_{s}^{I}(t)\right)
$$$$
\leqslant\left(e^{R}(t)\right)^{T}\left(I_{N} \otimes \tilde{D}^{I}\right) e^{R}(t)+\left(e^{I}(t)\right)^{T}\left(I_{N} \otimes L^{I}\right) e^{I}(t),
$$

$$
2 \sum_{s=1}^{N}\left(e_{s}^{I}(t)\right)^{T} D^{R}\left(Z_{s}^{R}(t)\right) P^{I}\left(e_{s}^{I}(t)\right)
$$$$
\leqslant\left(e^{I}(t)\right)^{T}\left(I_{N} \otimes \tilde{D}^{R}\right) e^{I}(t)+\left(e^{I}(t)\right)^{T}\left(I_{N} \otimes L^{I}\right) e^{I}(t),
$$

$$
2 \sum_{s=1}^{N}\left(e_{s}^{I}(t)\right)^{T} D^{I}\left(Z_{s}^{I}(t)\right) P^{R}\left(e_{s}^{R}(t)\right)
$$$$
\leqslant\left(e^{I}(t)\right)^{T}\left(I_{N} \otimes \tilde{D}^{I}\right) e^{I}(t)+\left(e^{R}(t)\right)^{T}\left(I_{N} \otimes L^{R}\right) e^{R}(t) .
$$

Moreover,

$$
\begin{aligned}
& 2 \sum_{s=1}^{N}\left(e_{s}^{R}(t)\right)^{T} B^{R}\left(Z_{s}^{R}(t)\right) Q^{R}\left(\overline{e_{s}^{R}(t)}\right) \\
\leqslant & \left(e^{R}(t)\right)^{T}\left(I_{N} \otimes \tilde{B}^{R}\right) e^{R}(t)+\left(\overline{e^{R}(t)}\right)^{T}\left(I_{N} \otimes \zeta^{R}\right) \overline{e^{R}(t)} .
\end{aligned}
$$

Similarly,

$$
-2 \sum_{s=1}^{N}\left(e_{s}^{R}(t)\right)^{T} B^{I}\left(Z_{s}^{I}(t)\right) Q^{I}\left(\overline{e_{s}^{I}(t)}\right)
$$

$\leqslant\left(e^{R}(t)\right)^{T}\left(I_{N} \otimes \tilde{B}^{I}\right) e^{R}(t)+\left(\overline{e^{I}(t)}\right)^{T}\left(I_{N} \otimes \zeta^{I}\right) \overline{e^{I}(t)}$,

$$
2 \sum_{s=1}^{N}\left(e_{s}^{I}(t)\right)^{T} B^{R}\left(Z_{s}^{R}(t)\right) Q^{I}\left(\overline{e_{s}^{I}(t)}\right)
$$$$
\leqslant\left(e^{I}(t)\right)^{T}\left(I_{N} \otimes \tilde{B}^{R}\right) e^{I}(t)+\left(\overline{e^{I}(t)}\right)^{T}\left(I_{N} \otimes \zeta^{I}\right) \overline{e^{I}(t)},
$$

$$
2 \sum_{s=1}^{N}\left(e_{s}^{I}(t)\right)^{T} B^{I}\left(Z_{s}^{I}(t)\right) Q^{R}\left(\overline{e_{s}^{R}(t)}\right)
$$

$\leqslant\left(e^{I}(t)\right)^{T}\left(I_{N} \otimes \tilde{B}^{I}\right) e^{I}(t)+\left(\overline{e^{R}(t)}\right)^{T}\left(I_{N} \otimes \zeta^{R}\right) \overline{e^{R}(t)}$. (17) 


$$
\begin{aligned}
= & g\left(e^{R}(t)\right)^{T}\left(G \otimes\left(M+M^{T}\right)\right) e^{R}(t), \\
& 2 g \sum_{s=1}^{N} \sum_{\kappa=1}^{N}\left(e_{s}^{I}(t)\right)^{T} G_{s \kappa} M e_{\kappa}^{I}(t) \\
= & g\left(e^{I}(t)\right)^{T}\left(G \otimes\left(M+M^{T}\right)\right) e^{I}(t) .
\end{aligned}
$$

What's more,

$$
\begin{aligned}
& 2 \sum_{s=1}^{N}\left(e_{s}^{R}(t)\right)^{T}\left(D^{R}\left(Z_{s}^{R}(t)\right)-D^{R}\left(Z_{0}^{R}(t)\right)\right) f^{R}\left(Z_{0}^{R}(t)\right) \\
\leqslant & 2 \sum_{s=1}^{N} \sum_{\iota=1}^{n} \sum_{j=1}^{n}\left|e_{s \iota}^{R}(t)\right|\left|\hat{d}_{\iota j}^{R}-\breve{d}_{\iota j}^{R}\right| F_{j}^{R} \\
= & 2 \sum_{s=1}^{N}\left|\left(e_{s}^{R}(t)\right)^{T}\right| \bar{D}^{R} \bar{F}^{R} .
\end{aligned}
$$

Similarly,

$$
\begin{aligned}
& 2 \sum_{s=1}^{N}\left(e_{s}^{R}(t)\right)^{T}\left(D^{I}\left(Z_{0}^{I}(t)\right)-D^{I}\left(Z_{s}^{I}(t)\right)\right) f^{I}\left(Z_{0}^{I}(t)\right) \\
\leqslant & 2 \sum_{s=1}^{N}\left|\left(e_{s}^{R}(t)\right)^{T}\right| \bar{D}^{I} \bar{F}^{I} \\
& 2 \sum_{s=1}^{N}\left(e_{s}^{I}(t)\right)^{T}\left(D^{R}\left(Z_{s}^{R}(t)\right)-D^{R}\left(Z_{0}^{R}(t)\right)\right) f^{I}\left(Z_{0}^{I}(t)\right) \\
\leqslant & 2 \sum_{s=1}^{N}\left|\left(e_{s}^{I}(t)\right)^{T}\right| \bar{D}^{R} \bar{F}^{I} \\
& 2 \sum_{s=1}^{N}\left(e_{s}^{I}(t)\right)^{T}\left(D^{I}\left(Z_{s}^{I}(t)\right)-D^{I}\left(Z_{0}^{I}(t)\right)\right) f^{R}\left(Z_{0}^{R}(t)\right) \\
\leqslant & 2 \sum_{s=1}^{N}\left|\left(e_{s}^{I}(t)\right)^{T}\right| \bar{D}^{I} \bar{F}^{R} \\
& 2 \sum_{s=1}^{N}\left(e_{s}^{R}(t)\right)^{T}\left(B^{R}\left(Z_{s}^{R}(t)\right)-B^{R}\left(Z_{0}^{R}(t)\right)\right) h^{R}\left(Z_{0}^{R}(t)\right) \\
\leqslant & 2 \sum_{s=1}^{N}\left|\left(e_{s}^{R}(t)\right)^{T}\right| \bar{B}^{R} \bar{H}^{R}, \\
& 2 \sum_{s=1}^{N}\left(e_{s}^{R}(t)\right)^{T}\left(B^{I}\left(Z_{0}^{I}(t)\right)-B^{I}\left(Z_{s}^{I}(t)\right)\right) h^{I}\left(Z_{0}^{I}(t)\right) \\
\leqslant & 2 \sum_{s=1}^{N}\left|\left(e_{s}^{R}(t)\right)^{T}\right| \bar{B}^{I} \bar{H}^{I} \\
& 2 \sum_{s=1}^{N}\left(e_{s}^{I}(t)\right)^{T}\left(B^{R}\left(Z_{s}^{R}(t)\right)-B^{R}\left(Z_{0}^{R}(t)\right)\right) h^{I}\left(Z_{0}^{I}(t)\right) \\
\leqslant & 2 \sum_{s=1}^{N}\left|\left(e_{s}^{I}(t)\right)^{T}\right| \bar{B}^{R} \bar{H}^{I} \\
& 2 \sum_{s=1}^{N}\left(e_{s}^{I}(t)\right)^{T}\left(B^{I}\left(Z_{s}^{I}(t)\right)-B^{I}\left(Z_{0}^{I}(t)\right)\right) h^{R}\left(Z_{0}^{R}(t)\right) \\
&
\end{aligned}
$$

Eqs. (9)-(27) yield

$$
\begin{aligned}
\dot{V}(t) \leqslant & \left(e^{R}(t)\right)^{T}\left(I _ { N } \otimes \left(-2 A+\tilde{D}^{R}+2 L^{R}+\tilde{D}^{I}+\tilde{B}^{R}+\tilde{B}^{I}-\Upsilon^{R}\right.\right. \\
& \left.\left.+2 \zeta^{R} \Gamma\right)+g G \otimes\left(M+M^{T}\right)\right) e^{R}(t)+\left(e^{I}(t)\right)^{T}\left(I_{N} \otimes(\right. \\
& \left.-2 A+2 L^{I}+\tilde{D}^{R}+\tilde{D}^{I}+\tilde{B}^{I}+\tilde{B}^{R}-\Upsilon^{I}+2 \zeta^{I} \Gamma\right) \\
& \left.+g G \otimes\left(M+M^{T}\right)\right) e^{I}(t)+2\left(e^{R}(t)\right)^{T} x^{R}(t) \\
& +2\left(e^{I}(t)\right)^{T} x^{I}(t) .
\end{aligned}
$$

Furthermore,

$$
\begin{aligned}
& \dot{V}(t)-\left[\left(y^{R}(t)\right)^{T} x^{R}(t)+\left(y^{I}(t)\right)^{T} x^{I}(t)\right] \\
& -\rho\left[\left(x^{R}(t)\right)^{T} x^{R}(t)+\left(x^{I}(t)\right)^{T} x^{I}(t)\right] \\
\leqslant & \left(\varphi^{R}(t)\right)^{T}\left(\begin{array}{ll}
\Psi_{1}^{R} & \Xi^{R} \\
\left(\Xi^{R}\right)^{T} & \Psi_{2}^{R}
\end{array}\right) \varphi^{R}(t) \\
& +\left(\varphi^{I}(t)\right)^{T}\left(\begin{array}{ll}
\Psi_{1}^{I} & \Xi^{I} \\
\left(\Xi^{I}\right)^{T} & \Psi_{2}^{I}
\end{array}\right) \varphi^{I}(t),
\end{aligned}
$$

where $\varphi^{R}(t)=\left(\left(e^{R}(t)\right)^{T},\left(x^{R}(t)\right)^{T}\right)^{T}$ and $\varphi^{I}(t)=$ $\left(\left(e^{I}(t)\right)^{T},\left(x^{I}(t)\right)^{T}\right)^{T}$. From (8), it is easy to obtain

$$
\begin{aligned}
\dot{V}(t) \leqslant & \left(y^{R}(t)\right)^{T} x^{R}(t)+\left(y^{I}(t)\right)^{T} x^{I}(t) \\
& +\rho\left[\left(x^{R}(t)\right)^{T} x^{R}(t)+\left(x^{I}(t)\right)^{T} x^{I}(t)\right] .
\end{aligned}
$$

By integrating (28) about $t$ over the time period from $t_{1}$ to $t_{2}$, one has

$$
\begin{aligned}
V\left(t_{2}\right)-V\left(t_{1}\right) \leqslant & \int_{t_{1}}^{t_{2}}\left[\left(y^{R}(t)\right)^{T} x^{R}(t)+\left(y^{I}(t)\right)^{T} x^{I}(t)\right] d t \\
& +\rho \int_{t_{1}}^{t_{2}}\left[\left(x^{R}(t)\right)^{T} x^{R}(t)+\left(x^{I}(t)\right)^{T} x^{I}(t)\right] d t,
\end{aligned}
$$

where $t_{2} \geqslant t_{1}$. Namely,

$$
\begin{aligned}
& \int_{t_{1}}^{t_{2}}\left[\left(y^{R}(t)\right)^{T} x^{R}(t)+\left(y^{I}(t)\right)^{T} x^{I}(t)\right] d t \geqslant V\left(t_{2}\right)-V\left(t_{1}\right) \\
& -\rho \int_{t_{1}}^{t_{2}}\left[\left(x^{R}(t)\right)^{T} x^{R}(t)+\left(x^{I}(t)\right)^{T} x^{I}(t)\right] d t
\end{aligned}
$$

for any $t_{2}, t_{1} \in \mathbb{R}^{+}$and $t_{2} \geqslant t_{1}$.

According to Definition III.1, we can obtain that the network (6) is passive under the controller (7).

C. Synchronization control

Theorem III.2. The network (2) is synchronized under the controller (7) if

$$
\Psi_{1}^{R}<0 \text { and } \Psi_{1}^{I}<0,
$$

where $\Psi_{1}^{R}=I_{N} \otimes\left(-2 A+\tilde{D}^{R}+2 L^{R}+\tilde{D}^{I}+\tilde{B}^{R}+\tilde{B}^{I}-\right.$ $\left.\Upsilon^{R}+2 \zeta^{R} \Gamma\right)+g G \otimes\left(M+M^{T}\right), \Psi_{1}^{I}=I_{N} \otimes\left(-2 A+2 L^{I}+\right.$ $\left.\tilde{D}^{R}+\tilde{D}^{I}+\tilde{B}^{I}+\tilde{B}^{R}-\Upsilon^{I}+2 \zeta^{I} \Gamma\right)+g G \otimes\left(M+M^{T}\right)$.

Proof. We construct the same Lyapunov functional as in Theorem III.1 in this subsection. Then, one obtains

$$
\begin{aligned}
\dot{V}(t) \leqslant & \left(e^{R}(t)\right)^{T}\left(I _ { N } \otimes \left(-2 A+\tilde{D}^{R}+2 L^{R}+\tilde{D}^{I}+\tilde{B}^{R}+\tilde{B}^{I}\right.\right. \\
& \left.\left.-\Upsilon^{R}+2 \zeta^{R} \Gamma\right)+g G \otimes\left(M+M^{T}\right)\right) e^{R}(t) \\
& +\left(e^{I}(t)\right)^{T}\left(I _ { N } \otimes \left(-2 A+2 L^{I}+\tilde{D}^{R}+\tilde{D}^{I}+\tilde{B}^{I}+\tilde{B}^{R}\right.\right.
\end{aligned}
$$




$$
\begin{aligned}
& \left.\left.-\Upsilon^{I}+2 \zeta^{I} \Gamma\right)+g G \otimes\left(M+M^{T}\right)\right) e^{I}(t) \\
\leqslant & \alpha\|e(t)\|^{2},
\end{aligned}
$$

where $\alpha=\max \left\{\lambda_{M}\left(\Psi_{1}^{R}\right), \lambda_{M}\left(\Psi_{1}^{I}\right)\right\}$.

According to (30) and the definition of $V(t)$, we can obtain $V(t)$ is non-increasing and bounded. Hence, $\lim _{t \rightarrow+\infty} V(t)$ exists and satisfies $\lim _{t \rightarrow+\infty} V(t) \geqslant 0$. In addition, from (30), we can get

$$
\|e(t)\|^{2} \leqslant \frac{\dot{V}(t)}{\alpha} .
$$

From (31), it is easy to derive that $\lim _{t \rightarrow+\infty} \int_{0}^{t}\|e(\delta)\|^{2} d \delta$ exists and is a nonnegative real number. Moreover,

$$
\begin{aligned}
0 & \leqslant \lim _{t \rightarrow+\infty} \sum_{s=1}^{N} \sum_{j=1}^{n} \int_{t-\tau_{j}(t)}^{t} \frac{2\left(\eta_{j}^{R} e_{s j}^{R}(\delta)\right)^{2}}{1-\gamma_{j}} d \delta \\
& \leqslant \lim _{t \rightarrow+\infty} \int_{t-\tau}^{t}\left(e^{R}(\delta)\right)^{T}\left(I_{N} \otimes\left(2 \zeta^{R} \Gamma\right)\right) e^{R}(\delta) d \delta \\
& \leqslant \lambda_{M}\left(I_{N} \otimes\left(2 \zeta^{R} \Gamma\right)\right) \lim _{t \rightarrow+\infty} \int_{t-\tau}^{t}\left\|e^{R}(\delta)\right\|^{2} d \delta \\
& =0 .
\end{aligned}
$$

Similarly,

$$
0 \leqslant \lim _{t \rightarrow+\infty} \sum_{s=1}^{N} \sum_{j=1}^{n} \int_{t-\tau_{j}(t)}^{t} \frac{2\left(\eta_{j}^{I} e_{s j}^{I}(\delta)\right)^{2}}{1-\gamma_{j}} d \delta=0 .
$$

From (32) and (33), we can easily know that $\lim _{t \rightarrow+\infty} \sum_{s=1}^{N}\left[\left(e_{s}^{R}(t)\right)^{T} e_{s}^{R}(t)+\left(e_{s}^{I}(t)\right)^{T} e_{s}^{I}(t)\right]$ exists and is a nonnegative real number. Suppose that

$$
\lim _{t \rightarrow+\infty} \sum_{s=1}^{N}\left[\left(e_{s}^{R}(t)\right)^{T} e_{s}^{R}(t)+\left(e_{s}^{I}(t)\right)^{T} e_{s}^{I}(t)\right]=\beta>0 .
$$

Then, there exists a real number $\epsilon>0$ satisfying

$$
\sum_{s=1}^{N}\left[\left(e_{s}^{R}(t)\right)^{T} e_{s}^{R}(t)+\left(e_{s}^{I}(t)\right)^{T} e_{s}^{I}(t)\right]>\frac{\beta}{2} \text { for } t \geqslant \epsilon .
$$

Then, one has

$$
\|e(t)\|^{2}>\frac{\beta}{2}, \quad t \geqslant \epsilon .
$$

Combined (30) and (34), one has

$$
\dot{V}(t)<\frac{\alpha \beta}{2}, \quad t \geqslant \epsilon .
$$

By (35), we can acquire

$-V(\epsilon) \leqslant V(+\infty)-V(\epsilon)=\int_{\epsilon}^{+\infty} \dot{V}(t) d t<\int_{\epsilon}^{+\infty} \frac{\alpha \beta}{2} d t=-\infty$, which is unreasonable. Therefore,

$$
\lim _{t \rightarrow+\infty} \sum_{s=1}^{N}\left[\left(e_{s}^{R}(t)\right)^{T} e_{s}^{R}(t)+\left(e_{s}^{I}(t)\right)^{T} e_{s}^{I}(t)\right]=0 .
$$

Then, we can obtain

$$
\lim _{t \rightarrow+\infty}\|e(t)\|=0 .
$$

Consequently, the network (2) achieves synchronization.

\section{NUMERICAL EXAMPLES}

Example IV.1. Consider the following CCVMNN:

$$
\begin{aligned}
\dot{Z}_{s}(t)= & -A Z_{s}(t)+B\left(Z_{s}(t)\right) h\left(\overline{Z_{s}(t)}\right)+u_{s}(t)+x_{s}(t) \\
& +D\left(Z_{s}(t)\right) f\left(Z_{s}(t)\right)+g \sum_{\kappa=1}^{N} G_{s \kappa} M Z_{\kappa}(t),
\end{aligned}
$$

where $s=1,2, \cdots, 6, f_{i}^{R}(\omega)=f_{i}^{I}(\omega)=h_{i}^{R}(\omega)=h_{i}^{I}(\omega)=$ $\frac{|\omega+1|-|\omega-1|}{4}(i=1,2,3), A=\operatorname{diag}(1.3,0.8,1.2), \quad M=$ $\operatorname{diag}(0.5,0.4,0.6), g=0.3, \tau_{j}(t)=1-\frac{1}{2+j} e^{-t}, \tau=1, \gamma_{j}=$ $\frac{1}{2+j}, j=1,2,3$, and the matrices $B\left(Z_{s}(t)\right), D\left(Z_{s}(t)\right), G=$ $\left(G_{s \kappa}\right)_{6 \times 6}$ are selected as follows:

$$
\begin{aligned}
& b_{11}^{R}\left(z_{s 1}^{R}(t)\right)=\left\{\begin{aligned}
0.36, & \left|z_{s 1}^{R}(t)\right| \leqslant 1.5, \\
-0.28, & \left|z_{s 1}^{R}(t)\right|>1.5,
\end{aligned}\right. \\
& b_{12}^{R}\left(z_{s 1}^{R}(t)\right)= \begin{cases}-0.25, & \left|z_{s 1}^{R}(t)\right| \leqslant 1.5, \\
-0.42, & \left|z_{s 1}^{R}(t)\right|>1.5,\end{cases} \\
& b_{13}^{R}\left(z_{s 1}^{R}(t)\right)=\left\{\begin{aligned}
-0.22, & \left|z_{s 1}^{R}(t)\right| \leqslant 1.5, \\
0.33, & \left|z_{s 1}^{R}(t)\right|>1.5,
\end{aligned}\right. \\
& b_{21}^{R}\left(z_{s 2}^{R}(t)\right)=\left\{\begin{aligned}
0.33, & \left|z_{s 2}^{R}(t)\right| \leqslant 1.5, \\
-0.27, & \left|z_{s 2}^{R}(t)\right|>1.5,
\end{aligned}\right. \\
& b_{22}^{R}\left(z_{s 2}^{R}(t)\right)= \begin{cases}0.26, & \left|z_{s 2}^{R}(t)\right| \leqslant 1.5, \\
0.25, & \left|z_{s 2}^{R}(t)\right|>1.5,\end{cases} \\
& b_{23}^{R}\left(z_{s 2}^{R}(t)\right)=\left\{\begin{aligned}
0.18, & \left|z_{s 2}^{R}(t)\right| \leqslant 1.5, \\
-0.14, & \left|z_{s 2}^{R}(t)\right|>1.5,
\end{aligned}\right. \\
& b_{31}^{R}\left(z_{s 3}^{R}(t)\right)=\left\{\begin{aligned}
0.25, & \left|z_{s 3}^{R}(t)\right| \leqslant 1.5, \\
-0.45, & \left|z_{s 3}^{R}(t)\right|>1.5,
\end{aligned}\right. \\
& b_{32}^{R}\left(z_{s 3}^{R}(t)\right)=\left\{\begin{aligned}
-0.34, & \left|z_{s 3}^{R}(t)\right| \leqslant 1.5, \\
0.25, & \left|z_{s 3}^{R}(t)\right|>1.5,
\end{aligned}\right. \\
& b_{33}^{R}\left(z_{s 3}^{R}(t)\right)= \begin{cases}0.17, & \left|z_{s 3}^{R}(t)\right| \leqslant 1.5, \\
0.21, & \left|z_{s 3}^{R}(t)\right|>1.5,\end{cases} \\
& b_{11}^{I}\left(z_{s 1}^{I}(t)\right)=\left\{\begin{aligned}
-0.35, & \left|z_{s 1}^{I}(t)\right| \leqslant 1.5, \\
0.27, & \left|z_{s 1}^{I}(t)\right|>1.5,
\end{aligned}\right. \\
& b_{12}^{I}\left(z_{s 1}^{I}(t)\right)= \begin{cases}0.26, & \left|z_{s 1}^{I}(t)\right| \leqslant 1.5, \\
0.16, & \left|z_{s 1}^{I}(t)\right|>1.5,\end{cases} \\
& b_{13}^{I}\left(z_{s 1}^{I}(t)\right)=\left\{\begin{aligned}
-0.22, & \left|z_{s 1}^{I}(t)\right| \leqslant 1.5, \\
0.13, & \left|z_{s 1}^{I}(t)\right|>1.5,
\end{aligned}\right. \\
& b_{21}^{I}\left(z_{s 2}^{I}(t)\right)=\left\{\begin{aligned}
-0.32, & \left|z_{s 2}^{I}(t)\right| \leqslant 1.5, \\
0.26, & \left|z_{s 2}^{I}(t)\right|>1.5,
\end{aligned}\right. \\
& b_{22}^{I}\left(z_{s 2}^{I}(t)\right)= \begin{cases}0.34, & \left|z_{s 2}^{I}(t)\right| \leqslant 1.5, \\
0.12, & \left|z_{s 2}^{I}(t)\right|>1.5,\end{cases} \\
& b_{23}^{I}\left(z_{s 2}^{I}(t)\right)= \begin{cases}0.12, & \left|z_{s 2}^{I}(t)\right| \leqslant 1.5, \\
0.11, & \left|z_{s 2}^{I}(t)\right|>1.5,\end{cases} \\
& b_{31}^{I}\left(z_{s 3}^{I}(t)\right)=\left\{\begin{aligned}
0.25, & \left|z_{s 3}^{I}(t)\right| \leqslant 1.5, \\
-0.33, & \left|z_{s 3}^{I}(t)\right|>1.5,
\end{aligned}\right.
\end{aligned}
$$




$$
\begin{aligned}
& b_{32}^{I}\left(z_{s 3}^{I}(t)\right)=\left\{\begin{aligned}
0.14, & \left|z_{s 3}^{I}(t)\right| \leqslant 1.5, \\
-0.25, & \left|z_{s 3}^{I}(t)\right|>1.5,
\end{aligned}\right. \\
& b_{33}^{I}\left(z_{s 3}^{I}(t)\right)= \begin{cases}0.31, & \left|z_{s 3}^{I}(t)\right| \leqslant 1.5, \\
0.25, & \left|z_{s 3}^{I}(t)\right|>1.5,\end{cases} \\
& d_{11}^{R}\left(z_{s 1}^{R}(t)\right)= \begin{cases}-0.36, & \left|z_{s 1}^{R}(t)\right| \leqslant 1.5, \\
-0.15, & \left|z_{s 1}^{R}(t)\right|>1.5,\end{cases} \\
& d_{12}^{R}\left(z_{s 1}^{R}(t)\right)= \begin{cases}-0.26, & \left|z_{s 1}^{R}(t)\right| \leqslant 1.5, \\
-0.25, & \left|z_{s 1}^{R}(t)\right|>1.5,\end{cases} \\
& d_{13}^{R}\left(z_{s 1}^{R}(t)\right)=\left\{\begin{aligned}
0.24, & \left|z_{s 1}^{R}(t)\right| \leqslant 1.5, \\
-0.13, & \left|z_{s 1}^{R}(t)\right|>1.5,
\end{aligned}\right. \\
& d_{21}^{R}\left(z_{s 2}^{R}(t)\right)= \begin{cases}0.13, & \left|z_{s 2}^{R}(t)\right| \leqslant 1.5, \\
0.25, & \left|z_{s 2}^{R}(t)\right|>1.5,\end{cases} \\
& d_{22}^{R}\left(z_{s 2}^{R}(t)\right)= \begin{cases}-0.25, & \left|z_{s 2}^{R}(t)\right| \leqslant 1.5, \\
-0.17, & \left|z_{s 2}^{R}(t)\right|>1.5,\end{cases} \\
& d_{23}^{R}\left(z_{s 2}^{R}(t)\right)=\left\{\begin{aligned}
-0.12, & \left|z_{s 2}^{R}(t)\right| \leqslant 1.5, \\
0.23, & \left|z_{s 2}^{R}(t)\right|>1.5,
\end{aligned}\right. \\
& d_{31}^{R}\left(z_{s 3}^{R}(t)\right)=\left\{\begin{aligned}
-0.25, & \left|z_{s 3}^{R}(t)\right| \leqslant 1.5, \\
0.27, & \left|z_{s 3}^{R}(t)\right|>1.5,
\end{aligned}\right. \\
& d_{32}^{R}\left(z_{s 3}^{R}(t)\right)= \begin{cases}0.23, & \left|z_{s 3}^{R}(t)\right| \leqslant 1.5, \\
0.11, & \left|z_{s 3}^{R}(t)\right|>1.5,\end{cases} \\
& d_{33}^{R}\left(z_{s 3}^{R}(t)\right)= \begin{cases}-0.13, & \left|z_{s 3}^{R}(t)\right| \leqslant 1.5, \\
-0.14, & \left|z_{s 3}^{R}(t)\right|>1.5,\end{cases} \\
& d_{11}^{I}\left(z_{s 1}^{I}(t)\right)= \begin{cases}-0.16, & \left|z_{s 1}^{I}(t)\right| \leqslant 1.5, \\
-0.26, & \left|z_{s 1}^{I}(t)\right|>1.5,\end{cases} \\
& d_{12}^{I}\left(z_{s 1}^{I}(t)\right)= \begin{cases}0.26, & \left|z_{s 1}^{I}(t)\right| \leqslant 1.5, \\
0.15, & \left|z_{s 1}^{I}(t)\right|>1.5,\end{cases} \\
& d_{13}^{I}\left(z_{s 1}^{I}(t)\right)=\left\{\begin{aligned}
0.21, & \left|z_{s 1}^{I}(t)\right| \leqslant 1.5, \\
-0.13, & \left|z_{s 1}^{I}(t)\right|>1.5,
\end{aligned}\right. \\
& d_{21}^{I}\left(z_{s 2}^{I}(t)\right)= \begin{cases}0.22, & \left|z_{s 2}^{I}(t)\right| \leqslant 1.5, \\
0.28, & \left|z_{s 2}^{I}(t)\right|>1.5,\end{cases} \\
& d_{22}^{I}\left(z_{s 2}^{I}(t)\right)=\left\{\begin{aligned}
-0.24, & \left|z_{s 2}^{I}(t)\right| \leqslant 1.5, \\
0.16, & \left|z_{s 2}^{I}(t)\right|>1.5,
\end{aligned}\right. \\
& d_{23}^{I}\left(z_{s 2}^{I}(t)\right)=\left\{\begin{aligned}
0.22, & \left|z_{s 2}^{I}(t)\right| \leqslant 1.5, \\
-0.11, & \left|z_{s 2}^{I}(t)\right|>1.5,
\end{aligned}\right. \\
& d_{31}^{I}\left(z_{s 3}^{I}(t)\right)= \begin{cases}-0.11, & \left|z_{s 3}^{I}(t)\right| \leqslant 1.5, \\
-0.16, & \left|z_{s 3}^{I}(t)\right|>1.5,\end{cases} \\
& d_{32}^{I}\left(z_{s 3}^{I}(t)\right)=\left\{\begin{aligned}
-0.24, & \left|z_{s 3}^{I}(t)\right| \leqslant 1.5, \\
0.11, & \left|z_{s 3}^{I}(t)\right|>1.5,
\end{aligned}\right. \\
& d_{33}^{I}\left(z_{s 3}^{I}(t)\right)=\left\{\begin{aligned}
-0.34, & \left|z_{s 3}^{I}(t)\right| \leqslant 1.5, \\
0.25, & \left|z_{s 3}^{I}(t)\right|>1.5,
\end{aligned}\right.
\end{aligned}
$$

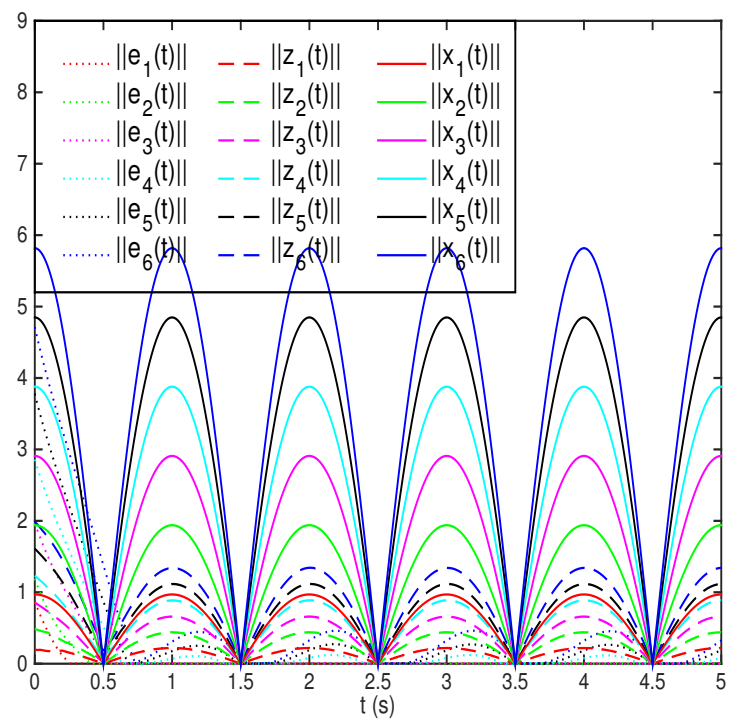

Fig. 1. The norms of $e_{s}(t), z_{s}(t), x_{s}(t), s=1,2, \cdots, 6$.

$$
H=\left(\begin{array}{cccccc}
-0.6 & 0.2 & 0.1 & 0 & 0.2 & 0.1 \\
0.2 & -0.7 & 0.1 & 0 & 0.3 & 0.1 \\
0.1 & 0.1 & -0.9 & 0.1 & 0.5 & 0.1 \\
0 & 0 & 0.1 & -0.4 & 0.2 & 0.1 \\
0.2 & 0.3 & 0.5 & 0.2 & -1.4 & 0.2 \\
0.1 & 0.1 & 0.1 & 0.1 & 0.2 & -0.6
\end{array}\right)
$$

Obviously, $f_{i}^{R}(\cdot), f_{i}^{I}(\cdot), h_{i}^{R}(\cdot)$, and $h_{i}^{I}(\cdot)(i=1,2,3)$ satisfy Assumption 1 with $F_{i}^{R}=F_{i}^{I}=H_{s}^{R}=H_{s}^{I}=0.5$ and $l_{s}^{R}=l_{s}^{I}=\eta_{s}^{R}=\eta_{s}^{I}=0.5$. The input $x_{s 1}(t)=$ $0.6 s \cos (t)+i 0.3 s \cos (t), x_{s 2}(t)=0.2 s \cos (t)+i 0.2 s \cos (t)$, $x_{s 3}(t)=0.4 s \cos (t)+i 0.5 s \cos (t)$. The parameters in the controller (7) are chosen as follows: $\Upsilon^{R}=\operatorname{diag}(0.9,1.2,0.6)$, $\Upsilon^{I}=\operatorname{diag}(0.8,1.0,0.9)$. Take $W_{1}$ and $W_{2}$ as follows:

$W_{1}=\left(\begin{array}{ccc}0 & 0.4 & 0 \\ 0.2 & -0.5 & 0.2 \\ 0.3 & 0 & -0.2\end{array}\right), W_{2}=\left(\begin{array}{ccc}-0.1 & 0.2 & 0.3 \\ 0 & -0.4 & 0.1 \\ 0.2 & 0.5 & -0.3\end{array}\right)$.

By using the MATLAB, it is easy to obtain that $\rho=3.7220$ which satisfies the condition (8). On the basis of Theorem III.1, the system (36) is passive under controller (7). Fig. 1 shows the evolutions of error, output and input of six nodes when the system (36) is passive. Similarly, through a simple operation based on the above parameters by utilizing the MATLAB, we can obtain

$$
\begin{aligned}
\lambda\left(\Psi_{1}^{R}\right)= & \{-1.6951,-1.5403,-1.4313,-1.4060,-1.3738, \\
& -1.2863,-1.2558,-1.2236,-1.1933,-1.1625, \\
& -1.1547,-1.0496,-1.0448,-1.0245,-0.9988, \\
& -0.9288,-0.9011,-0.8297\}, \\
\lambda\left(\Psi_{1}^{I}\right)= & \{-1.8403,-1.5951,-1.5236,-1.4933,-1.4547, \\
& -1.3496,-1.3313,-1.3060,-1.2738,-1.2011, \\
& -1.1863,-1.0625,-1.0558,-0.8448,-0.8245,
\end{aligned}
$$




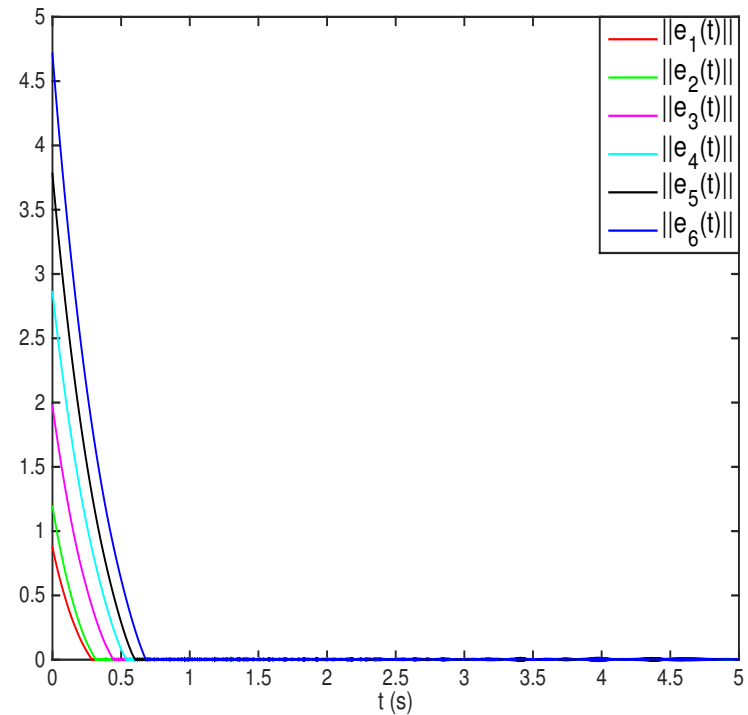

Fig. 2. Time evolutions of $e_{s}(t), s=1,2, \cdots, 6$.

$$
-0.7988,-0.7288,-0.6297\}
$$

which satisfy the condition (29). According to Theorem III.2, the system (36) achieves synchronization. Fig. 2 depicts the simulation result of synchronization.

\section{CONCLUSION}

This study has concerned with a type of CCVMNNs. By using certain inequalities, Lyapunov functionals as well as the design of suitable controller, a novel criterion for ensuring passivity of the considered network has been derived. Similarly, we have also carried out some discussion on the synchronization of CCVMNNs. A simulation example has been performed to confirm the correctness of our results at the end.

\section{REFERENCES}

[1] H. Lü, W. L. He, Q. L. Han, and C. Peng, "Fixed-time pinning-controlled synchronization for coupled delayed neural networks with discontinuous activations," Neural Netw., vol. 116, pp. 139 - 149, August 2019.

[2] A. Chandrasekar, R. Rakkiyappan, and J. Cao, "Impulsive synchronization of Markovian jumping randomly coupled neural networks with partly unknown transition probabilities via multiple integral approach," Neural Netw., vol. 70, pp. 27 - 38, October 2015.

[3] W. Wu and T. Chen, "Global synchronization criteria of linearly coupled neural network systems with time-varying coupling," IEEE Trans. Neural Netw., vol. 19, pp. 319 - 332, Februrary 2008.

[4] S. R. Lin, Y. L. Huang, and S. Y. Ren, "Analysis and pinning control for passivity of coupled different dimensional neural networks," Neurocomputing, vol. 321, pp. 187 - 200, December 2018.

[5] S. Y. Ren, J. L. Wang, and J. G. Wu, "Generalized passivity of coupled neural networks with directed and undirected topologies," Neurocomputing, vol. 314, pp. 371 - 385, November 2018.

[6] L. O. Chua, "Memristor-the missing circuit element," IEEE Trans. Circuit Theory, vol. 18, pp. 507 - 519, September 1971.

[7] H. Shen, T. Wang, J. D. Cao, G. P. Lu, Y. D. Song, and T. W. Huang, "Non-Fragile dissipative synchronization for markovian memristive neural networks: a gain-scheduled control scheme," IEEE Trans. Neural Netw. Learn. Syst., vol. 30, pp. 1841 - 1853, June 2019.
[8] L. M. Wang, Z. G. Zeng, M. F. Ge, and J. H. Hu, "Global stabilization analysis of inertial memristive recurrent neural networks with discrete and distributed delays," Neural Netw., vol. 105, pp. 65 - 74, September 2018.

[9] L. M. Wang, Y. Shen, and G. D. Zhang, "Finite-time stabilization and adaptive control of memristor-based delayed neural networks," IEEE Trans. Neural Netw. Learn. Syst., vol. 28, pp. 2648 - 2659, November 2017.

[10] Q. Xiao and Z. G. Zeng, "Lagrange stability for T-S fuzzy memristive neural networks with time-varying delays on time scales," IEEE Trans. Fuzzy Syst., vol. 26, pp. 1091-1103, June 2018.

[11] Y. L. Huang, S. H. Qiu and S. Y. Ren, "Finite-time synchronization and passivity of coupled memristive neural networks," Int. J. Control., doi: 10.1080/00207179.2019.1566640, January 2019.

[12] Y. V. Pershin and M. D. Ventra, "Experimental demonstration of associative memory with memristive neural networks," Neural Netw., vol. 23, pp. 881 - 886, September 2010.

[13] S. F. Yang, Z. Y. Guo, and J. Wang, "Robust synchronization of multiple memristive neural networks with uncertain parameters via nonlinear coupling," IEEE Trans. Syst. Man Cyber. Syst., vol. 45, pp. 1077 - 1086 July 2015.

[14] Z. Y. Guo, S. Q. Gong, S. F. Yang, and T. W. Huang, "Global exponential synchronization of multiple coupled inertial memristive neural networks with time-varying delay via nonlinear coupling," Neural Netw., vol. 108, pp. 260 - 271, December 2018.

[15] Z. Y. Guo, S. F. Yang, and J. Wang, "Global exponential synchronization of multiple memristive neural networks with time delay via nonlinear coupling," IEEE Trans. Neural Netw. Learn. Syst., vol. 26, pp. 1300 1311, June 2015.

[16] Y. F. Yuan, Q. K. Song, Y. R. Liu, and F. E. Alsaadi, "Synchronization of complex-valued neural networks with mixed two additive time-varying delays," Neurocomputing, vol. 332, pp. 149 - 158, March 2019.

[17] S. Yang, J. Yu, C. Hu, and H. J. Jiang, "Quasi-projective synchronization of fractional-order complex-valued recurrent neural networks," Neural Netw., vol. 104, pp. 104 - 113, August 2018.

[18] Y. Kan, J. Q. Lu, J. L. Qiu, and J. G. Kurths, "Exponential synchronization of time-varying delayed complex-valued neural networks under hybrid impulsive controllers," Neural Netw., vol. 114, pp. 157 - 163, June 2019.

[19] H. Zhang, X. Y. Wang, and X. H. Lin, "Synchronization of complexvalued neural network with sliding mode control," J. Frankl. Inst., vol. 353, pp. 345 - 358, January 2016.

[20] H. M. Wang, S. K. Duan, T. W. Huang, L. D. Wang, and C. D. Li, "Exponential stability of complex-Valued memristive recurrent neural networks," IEEE Trans. Neural Netw. Learn. Syst., vol. 28, pp. 766 771, March 2017.

[21] W. W. Zhang, H. Zhang, J. D. Cao, Fuad E. Alsaadi, and D. Y. Chen, "Synchronization in uncertain fractional-order memristive complexvalued neural networks with multiple time delays," Neural Netw., vol. 110, pp. 186 - 198, February 2019.

[22] R. Rakkiyappan, K. Sivaranjani, and G. Velmurugan, "Passivity and passification of memristor-based complex-valued recurrent neural networks with interval time-varying delays," Neurocomputing, vol. 144, pp. 391 407, November 2014.

[23] X. D. Li, R. Rakkiyappan, and G. Velmurugan, "Dissipativity analysis of memristor-based complex-valued neural networks with time-varying delays,” Inf. Sci., vol. 294, pp. 645 - 665, January 2018. 